\title{
Special issue on Neural Computing and Applications in cyber intelligence: ATCI 2019
}

\author{
Zheng $\mathrm{Xu}^{1} \cdot$ Jemal Abawajy ${ }^{2}$
}

Published online: 31 March 2020

(c) Springer-Verlag London Ltd., part of Springer Nature 2020

This special issue focuses on all aspects on techniques and applications in cyber intelligence research. The purpose of this special issue is to provide a forum for presentation and discussion of innovative ideas, cutting-edge research results, and novel techniques, methods and applications on all aspects of cyber intelligence. It includes suggested papers from 2019 International Conference on Applications and Techniques in Cyber Intelligence (ATCI2019) June 22-24 2019 Huainan as well as an open call.

The submitted manuscripts were reviewed by experts from both academia and industry. After two rounds of reviewing, the highest quality manuscripts were accepted for this special issue. This special issue will be published by Neural Computing and Applications as special issues. Totally, 18 papers are suggested to EiC for acceptance. The selected papers are summarized as follows.

Zhang et al. [1] investigated the parameter estimation method of the no-arbitrage Nelson-Siegel model for the classical interest rate model and pointed out the shortcomings of the estimation method. Fan et al. [2] constructed a feature classification model for Alzheimer's disease based on AdaBoost algorithm and KPCA algorithm and selected 21 patients with Alzheimer's disease (AD). Wang et al. [3] proposed convolution operators for visual tracking based on spatial-temporal regularization. Hu et al. [4] put forward the basic-element theory and studied the intelligent knowledge representation method of the basicelement theory. Zhong and Zhou [5] studied the microfinance risk based on polygenic artificial neural network, and it provides theory and practice application for risk management of microcredit enterprises. Xiao et al. [6] analyzed

Zheng Xu

zhengxu@shu.edu.cn

1 Shanghai University, 99 Shangda Road, Shanghai 201142, China

2 School of Information Technology, Deakin University, 221 Burwood Highway, Burwood, VIC 3125, Australia the shortcomings of current stock market forecasting methods and standard support vector machines firstly; at the same time, based on this, a cumulative auto-regressive moving average is proposed, which combines the least squares support vector machine synthesis model (ARI-MALS-SVM) to make basic predictions for the stock market. In order to achieve accurate judgment and identification of group behaviors, the hierarchical deep network model is constructed by Qiao et al. [7] to judge the group behaviors. Hou et al. [8] proposed an online client algorithm based on machine learning algorithm for IoT unstructured big data analysis and used it in other big data analysis scenarios. Guo et al. [9] proposed an improved multi-way matching deep neural network model for fine-grained sentiment analysis, which jointly models the two tasks in one phase and improves current attention by directly capturing past attention in the multi-round alignment architecture, so as to prevent error propagation and attention deficiency problems. Wang et al. [10] proposed a pancreatic cancer diagnosis model based on the fusion of distribution estimation algorithm and genetic algorithm.

A novel groupwise registration approach is proposed by Zhang et al. [11] mapping the extracted features from multimodality images into the same latent space. Chen et al. [12] carried out the work from the two aspects of feature extraction and feature classification of the ground object and analyzed and studied the application and value of the polarimetric SAR system. Huang et al. [13] mainly adopted three common machine learning algorithms: BP neural network, extreme learning machine and support vector machine. In order to detect the behavior of egg breeders in self-breeding cages rapidly, a method of target location and behavior recognition based on visual images was proposed by Wang et al. [14]. Gao and Yuan [15] proposed a research on pavement object recognition based on machine learning, including analysis of key technologies of machine learning and optimization of related algorithms. GSP is used by Zhai [16] as an applicable 
method to non-intrusive appliance load monitoring (NILM). Wang et al. [17] proposed an embedded adaptive cross-modulation (EACM) method for few-shot learning which combines the information between support and query examples. By combining the dynamic gain and the self-adaptive neural network, an output feedback faulttolerant control method was proposed by Xi et al. [18] for a class of nonlinear uncertain systems with actuator faults.

Acknowledgments The guest editors would like to thank Prof. John MacIntyre who is the editor in chief of Neural Computing and Applications. His help and trust are the most important thing for the success of this SI. The guest editors would like to thank the reviewers for their high-quality reviews, which provided insightful and constructive feedback to the authors of the papers.

\section{References}

1. Zhang Q, Wang X, Zhou X et al (2020) Application of improved adaptive Kalman filter in China's interest rate market. Neural Comput Appl. https://doi.org/10.1007/s00521-020-04706-Z

2. Fan Z, Xu F, Li C et al (2020) Application of KPCA and AdaBoost algorithm in classification of functional magnetic resonance imaging of Alzheimer's disease. Neural Comput Appl. https://doi.org/10.1007/s00521-020-04707-y

3. Wang P, Sun M, Wang $\mathrm{H}$ et al (2020) Convolution operators for visual tracking based on spatial-temporal regularization. Neural Comput Appl. https://doi.org/10.1007/s00521-020-04704-1

4. $\mathrm{Hu} \mathrm{R}$, Wang $\mathrm{H}, \mathrm{Xu} \mathrm{H}$ et al (2020) Research on intelligent knowledge representation method and algorithm based on basicelement theory. Neural Comput Appl. https://doi.org/10.1007/ s00521-020-04703-2

5. Zhong X, Zhou S (2020) Risk analysis method of bank microfinance based on multiple genetic artificial neural networks. Neural Comput Appl. https://doi.org/10.1007/s00521-019-04683-y

6. Xiao C, Xia W, Jiang J (2020) Stock price forecast based on combined model of ARI-MA-LS-SVM. Neural Comput Appl. https://doi.org/10.1007/s00521-019-04698-5

7. Qiao S, Wang L, Gao Z (2020) Group behavior recognition based on deep hierarchical network. Neural Comput Appl. https://doi. org/10.1007/s00521-019-04699-4
8. Hou R, Kong Y, Cai B et al (2020) Unstructured big data analysis algorithm and simulation of Internet of Things based on machine learning. Neural Comput Appl. https://doi.org/10.1007/s00521019-04682-z

9. Guo X, Zhang G, Wang S et al (2020) Multi-way matching based fine-grained sentiment analysis for user reviews. Neural Comput Appl. https://doi.org/10.1007/s00521-019-04686-9

10. Wang X, Li X, Chen X et al (2019) Diagnosis model of pancreatic cancer based on fusion of distribution estimation algorithm and genetic algorithm. Neural Comput Appl. https://doi. org/10.1007/s00521-019-04684-x

11. Zhang Y, Lian J, Jia W et al (2019) Multimodality registration for ocular multispectral images via co-embedding. Neural Comput Appl. https://doi.org/10.1007/s00521-019-04685-w

12. Chen G, Wang L, Kamruzzaman MM (2019) Spectral classification of ecological spatial polarization SAR image based on target decomposition algorithm and machine learning. Neural Comput Appl. https://doi.org/10.1007/s00521-019-04624-9

13. Huang J, Ko K, Shu M et al (2019) Application and comparison of several machine learning algorithms and their integration models in regression problems. Neural Comput Appl. https://doi. org/10.1007/s00521-019-04644-5

14. Wang J, Wang N, Li L et al (2019) Real-time behavior detection and judgment of egg breeders based on YOLO v3. Neural Comput Appl. https://doi.org/10.1007/s00521-019-04645-4

15. Gao H, Yuan L (2019) Research on key technology of pavement object recognition based on machine learning. Neural Comput Appl. https://doi.org/10.1007/s00521-019-04643-6

16. Zhai M (2019) A new graph learning-based signal processing approach for non-intrusive load disaggregation with active power measurements. Neural Comput Appl. https://doi.org/10.1007/ s00521-019-04623-w

17. Wang P, Cheng J, Hao F et al (2019) Embedded adaptive crossmodulation neural network for few-shot learning. Neural Comput Appl. https://doi.org/10.1007/s00521-019-04605-y

18. Xi X, Liu T, Zhao J et al (2019) Output feedback fault-tolerant control for a class of nonlinear systems via dynamic gain and neural network. Neural Comput Appl. https://doi.org/10.1007/ s00521-019-04583-1

Publisher's Note Springer Nature remains neutral with regard to jurisdictional claims in published maps and institutional affiliations. 